\title{
A Prospective Observational Study to Determine the Predictors of Increased Number of Attempts at Labour Epidural Placement
}

\author{
Samina Ismail (1), Syed Amir Raza (1) \\ Department of Anaesthesiology, Aga Khan University, Karachi, Pakistan
}

Cite this article as: Ismail S, Raza SA. A Prospective Observational Study to Determine the Predictors of Increased Number of Attempts at Labour Epidural Placement. Turk J Anaesthesiol Reanim 2020; 48(5): 379-84.

\begin{abstract}
Objective: Multiple attempts at labour epidural placement result in patient discomfort and high incidence of complications. Identifying the factors that lead to more than one attempt would help anaesthesiologists prepare in advance such as getting expert help and additional equipment, patient counselling or planning alternative management.

Methods: This prospective observational study was conducted on 500 patients from July 2017 to June 2018 after obtaining approval from the institutional Ethics Review Committee. The study patients consisted of full-term parturient women who were admitted in the labour room suite of Aga Khan Hospital requesting for labour epidural and consented to participate in the study. A predesigned form was used to collect the following data: number of attempts at epidural insertion and factors such as patients' demographics, cervical dilatation, anatomical grading of spine according to visibility and palpation of spinous process and vertebral interspace, experience level of the anaesthesiologist, patient satisfaction and pain score during labour.

Results: The average age of the patients was $28.11 \pm 4.02$ years. The total number of epidural attempts varied between one and four; the median number of attempts was $1[\mathrm{IQR}=1-2]$. Anatomical grade of the spine was the only factor that was significantly associated with more than one attempt at epidural insertion $(\mathrm{p}=0.0005)$. Patient satisfaction was negatively associated with the number of attempts $(\mathrm{p}=0.04)$, but mean pain difference at different time points during the course of labour was not statistically significant between patients with one attempt and those with more than one attempt.

Conclusion: Determining the anatomical grade of the spine is the most reliable method for predicting a technically difficult neuraxial block that requires more than one attempt at epidural insertion.

Keywords: Difficulty, grade of spine, labour epidural, number of attempts, predictors
\end{abstract}

\section{Introduction}

Epidural analgesia is considered as the most effective analgesic option for labouring women $(1,2)$. However, epidural placement has been found to be the most difficult manual skill in anaesthesiology (3). It has been reported that multiple attempts at epidural placement may lead to patient discomfort, higher incidence of spinal haematoma, post-dural puncture headache and trauma of neural structures $(4,5)$.

Previous study has attempted to identify various factors as predictors of a technically difficult neuraxial block in a heterogeneous group of non-obstetric population (6). Pregnancy has been associated with difficult epidural placement, most probably due to weight gain and tissue oedema with obscure anatomical landmarks, which can result in multiple attempts at epidural placement (7). The rationale of this study is to identify the factors that lead to more than one attempt at labour epidural placement in full-term parturient women requesting for labour epidural. An accurate prediction of difficulty at labour epidural placement would help anaesthesiologists prepare in advance such as patient counselling, getting experienced help and imaging equipment such as an ultrasound (8). This can be helpful in making this technique less traumatic and risky for this vulnerable group of patients. 
The primary objective of the present study is to determine as which identified factors are the best predictors for difficult epidural placement in obstetric patients, that can lead to more than one attempt at labour epidural placement. The secondary objective is to determine the effect of the number of attempts on patient satisfaction and pain score at different time points during the course of labour.

\section{Methods}

Approval for conducting this prospective observational study was obtained from the Ethics Review Committee (proposal number 3642-Ane-ERC-15) of Aga Khan University. This study was conducted for a period of 1 year on 500 patients using the consecutive non-probability sampling method after obtaining written informed consent from the patient and verbal consent from the anaesthesiologist performing the epidural analgesia. Inclusion criteria were full-term parturient women who were admitted in the labour room suite of Aga Khan Hospital requesting for labour epidural and consented to participate in the study. Exclusion criteria were patients with any contraindication for labour epidural and the patient and/ or the anaesthesiologist not consenting to be included in the study. Labour epidural is performed according to the set protocol in the unit where the study was performed, but it is still at the discretion of the anaesthesiologist performing the epidural procedure. The plan of labour epidural was discussed with the anaesthesiologist performing the epidural procedure, and when there was any diversion from the routine protocol, patients were not included in the study. All labour epidural procedures to be performed were assessed for the eligibility of inclusion in the study by the research assistant, and those cases fulfilling the inclusion criteria were recruited into the study.

The primary endpoint of the study was to identify the factors that were associated with more than one attempt at labour epidural placement. An epidural insertion attempt was identified as advancement of the needle in an effort to enter the

\section{Main Points:}

- Pregnancy is associated with technically difficult epidural placement.

- Traumatic or multiple attempts at labour epidural increase patient discomfort and decrease the level of satisfaction.

- Identifying factors leading to multiple attempts at labour epidural placement would facilitate anaesthesiologist prepare in advance.

- Among all the factors that were examined in this study, anatomical grade of the spine was the most significant predictor leading to multiple attempts.

- Therefore examination of patients back to determine anatomical grade of the spine is the most reliable method for predicting a technically difficult epidural block. epidural space, and any manoeuvre such as withdrawal of the needle and reinsertion was not counted as an additional attempt. Any new puncture, whether at the same spinal level or a different spinal level, was considered as another attempt, but simple redirection of the needle without a new puncture was not considered as another attempt. The following data were collected using the predesigned data collection form by the research assistant: number of epidural attempts and factors such as patients' demographics; gestational age; cervical dilatation at the time of epidural placement; experience level of the anaesthesiologist performing the procedure; anatomical grade of the spine; patients' pain score before the epidural procedure and at 30 and $45 \mathrm{~min}$ and 2,4 and $6 \mathrm{~h}$ post-epidural insertion; need to resite the epidural; need to abandon the procedure; accidental dural tap and patient satisfaction with the labour epidural.

Anatomical grading of the spine was performed by anaesthesiologists with more than 2 years of experience and not performing the epidural procedure according to the scale described in the study by Faitot et al. (1) as follows: grade $1=$ spinous processes visible, grade $2=$ spinous processes easily palpable but not visible, grade $3=$ spinous processes not visible or easily palpable but interspace palpable and grade $4=$ spinous processes and interspace neither visible nor palpable. The anaesthesiologist performing the epidural procedure had more than 2 years of experience and was blinded to the observers' grading of the spine. The method of performing the epidural procedure in the patients included in the study was in accordance with the set protocol of labour epidural of the obstetric unit where the study was performed. The epidural procedure was performed with the patient sitting on one side of the bed with the neck, back and hips flexed and feet supported by a footrest. The hips were positioned with the weight distributed evenly between both sides, with an assistant standing facing the patient, helping to maintain the patient's position.

The epidural procedure was performed using a 16-gauge Tuohy needle (Portex, Smith Medical, Hythe, UK) and a loss of resistance to air/saline technique. Analgesia was achieved using $10-15 \mathrm{~mL}$ of $0.125 \%$ bupivacaine distributed into two aliquots. Subsequent analgesia was maintained by infusing $0.125 \%$ bupivacaine at a rate of $10-15 \mathrm{~mL}$ per hour with additional boluses of $5-10 \mathrm{~mL}$ of the same solution when the numeric rating scale (NRS) was more than 4 .

Pain score was measured using the NRS, with ' 0 ' considered as no pain and ' 10 ' considered as the worst imaginable pain. The NRS score was recorded before the insertion of epidural and then at 15 and $45 \mathrm{~min}$ and 24 and $6 \mathrm{~h}$ either by the research assistant or by the labour room nurse who were not a part of the study. Satisfaction score indicating the extent of satisfaction of the patient with the epidural procedure and 
its effects on her labour pains was recorded by the research assistant $12 \mathrm{~h}$ after the delivery using a five-point satisfaction score as follows: 1) perfect: the patient did not experience any pain at all, 2) very satisfied: helped relieve most of her pains, 3) satisfied: the epidural procedure relieved major pains, but she still experienced minor pains, 4) not satisfied: the epidural procedure helped only a bit and 5) poor: the epidural procedure did not help at all. Scores from 1 to 3 were considered as the patient being satisfied with the labour epidural analgesia, and scores from 4 to 5 were regarded as the patient being unsatisfied with the labour epidural analgesia.

The anaesthesiologists performing the epidural procedure were grouped according to the year of training as follows: year 2-3, year 4 and year $\geq 5$ (fellows, senior trainees and consultants). The 5-year residency programme in the institution where this study was conducted has a fixed competency list for the procedures performed for every year/level of residency; therefore, almost all residents of each year have the same level of experience and the number of epidural procedures performed.

\section{Statistical analysis}

Data were analysed using the IBM Statistical Package for Social Sciences, version 19 (IBM SPSS Corp.; Armonk, NY, USA). Mean and standard deviation were computed for age, gestational age, weight, height, BMI and mean pain score. Frequency and percentage were computed for qualitative variables. Cervical dilatation and BMI values were categorised, and chi-square test or Fisher's exact test was used to determine the association between factors (i.e. cervical dilatation, BMI, anatomical grade of the spine, experience level of the anaesthesiologist) and the number of epidural attempts. An independent samples $t$-test was used to compare the mean pain score at each time point between women with one epidural attempt and those with more than one attempt. $\mathrm{P} \leq 0.05$ was considered to indicate statistical significance.

\section{Results}

During the study period of 12 months, epidural procedures were performed on a total of 529 patients, of whom 519 fulfilled our inclusion criteria. These eligible patients were approached for written informed consent, and nine declined

\begin{tabular}{|l|c|}
\hline \multicolumn{2}{|l|}{ Table 1. Patients' demographics $(\mathbf{n}=500)$} \\
\hline Variables & Point estimate \\
\hline Age (years) & $28.11 \pm 4.02$ \\
Gestational age (weeks) & $38.17 \pm 1.38$ \\
Weight $(\mathrm{kg})$ & $73.77 \pm 12.26$ \\
Height $(\mathrm{cm})$ & $158.74 \pm 19.49$ \\
BMI $\left(\mathrm{kg} \mathrm{m}^{-2}\right)$ & $29.59 \pm 5.11$ \\
\hline
\end{tabular}

to provide the consent and 10 patients were excluded from the study due to incomplete data entry. Table 1 shows the patients' demographic characteristics. The total number of attempts at labour epidural placement varied between one and four; the median number of attempts was $1(\mathrm{IQR}=1-2)$. Labour epidural placement at first attempt was successfully achieved in $79.6 \%$ of patients.

Analysis of the association of different predictive factors for technically difficult labour epidural placement with the number of attempts at labour epidural placement revealed that anatomical grade of the spine was the only factor to be significantly associated with more than one attempt at epidural insertion (Table 2). Patients with anatomical grading 4 underwent statistically significant more attempts at epidural placement $(\mathrm{p}<0.0005)$ (Table 2). Patient satisfaction was found to be less with more than one attempt $(p=0.04)$ (Table 2). The mean pain score was high before the epidural procedure, but it was significantly reduced at $15-45 \mathrm{~min}$. However, there was no difference in the pain score between patients with one attempt and those with more than one attempt at epidural placement (Figure 1).

A total of $76(15.2 \%)$ epidural procedures were considered as failures by the investigators. These were caused due to the failure to relieve labour pain and the persistence of pain, with the NRS score being $>4$ after 45 min of administering the initial bolus of local anaesthetics and the initiation of epidural infusion in $39(8 \%)$ patients. The other factors responsible for the failures were the need to resite in $11(2.2 \%)$ patients, the need to abandon the procedure in $3(0.6 \%)$ patients, accidental dural puncture in $8(1.6 \%)$ patients and an overall dissatisfaction of patients with epidural pain relief in $55(11 \%)$ patients.

\section{Discussion}

Among central neuraxial blocks, epidural analgesia is considered to be a popular choice for labour analgesia; however, weight gain and tissue changes associated with pregnancy may lead to difficult epidural placement. To avoid traumatic needle placement, it is important to identify a priori the parturient women who may be at a greater risk of difficult epidural placement. Difficult central neuraxial block placement has been associated with complications as observed in six reports of spinal haematoma, where four cases of the six were termed as technically difficult (9).

Although anaesthesiologists have been using predictors of difficult airway (10), there is little evidence to help predict the difficulty at epidural insertion for labouring parturient women. In the present study, we attempted to identify the predictors of difficult labour epidural placement in full-term parturient 


\begin{tabular}{|c|c|c|c|c|}
\hline \multirow[b]{2}{*}{ Variables } & \multirow[b]{2}{*}{$\mathbf{n}$} & \multicolumn{2}{|c|}{ Number of attempts } & \multirow[b]{2}{*}{$\mathbf{p}$} \\
\hline & & $\begin{array}{c}1^{\text {st }} \text { attempts } \\
n=398\end{array}$ & $\begin{array}{c}2-4 \text { attempts } \\
n=102\end{array}$ & \\
\hline \multicolumn{5}{|c|}{ Cervical dilatation } \\
\hline$<3$ & 12 & $91(83.5 \%)$ & $18(16.5 \%)$ & 0.255 \\
\hline $3-5$ & 379 & $300(79.2 \%)$ & $79(20.8 \%)$ & 0.665 \\
\hline$>5$ & 109 & $7(58.3 \%)$ & $5(41.7 \%)$ & 0.076 \\
\hline \multicolumn{5}{|l|}{ BMI } \\
\hline$<25$ & 69 & $61(88.4 \%)$ & $8(11.6 \%)$ & 0.054 \\
\hline $25-29.9$ & 212 & $167(78.8 \%)$ & $45(21.2 \%)$ & 0.694 \\
\hline$\geq 30$ & 219 & $170(77.6 \%)$ & $49(22.4 \%)$ & 0.333 \\
\hline \multicolumn{5}{|l|}{ Grades of spine } \\
\hline Grade 1 & 41 & $34(82.9 \%)$ & $7(17.1 \%)$ & 0.581 \\
\hline Grade 2 & 344 & $289(84 \%)$ & $55(16 \%)$ & 0.0005 \\
\hline Grade 3 & 98 & $70(71.4 \%)$ & $28(28.6 \%)$ & 0.025 \\
\hline Grade 4 & 17 & $5(29.4 \%)$ & $12(70.6 \%)$ & 0.0005 \\
\hline \multicolumn{5}{|c|}{ Experience level of anaesthesiologist } \\
\hline $2^{\text {nd }}$ or $3^{\text {rd }}$ year & 190 & $146(76.8 \%)$ & $44(23.2 \%)$ & 0.231 \\
\hline $4^{\text {th }}$ year & 99 & $82(82.8 \%)$ & $17(17.2 \%)$ & 0.373 \\
\hline $5^{\text {th }}$ year and above & 211 & $170(80.6 \%)$ & $41(19.4 \%)$ & 0.646 \\
\hline \multicolumn{5}{|c|}{ Patient satisfaction } \\
\hline Satisfied & 445 & $360(80.9 \%)$ & $85(19.1 \%)$ & 0.040 \\
\hline
\end{tabular}

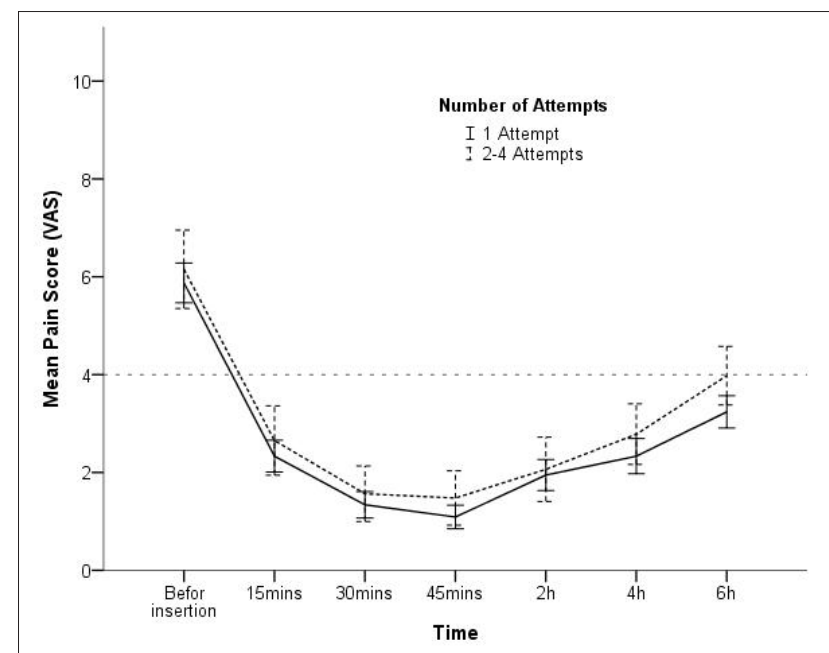

Figure 1. Mean pain score during the course of labour with respect to the number of epidural attempts

women. As these predictors can help identify parturient women with difficult epidural placement and instead of having traumatic epidural placement with multiple attempts, expert help can be sought or additional equipment such as an ultrasound can be arranged before attempting epidural placement. Furthermore, the patient can be counselled for a possible failure and the choice of an alternative technique for labour analgesia.
Among all the factors that were examined in this study, anatomical grade of the spine was the most significant predictor as measured by the number of attempts. The results of this study confirm those of previous studies $(3,6)$. However, there was no statistically significant association between the number of epidural attempts and the patients' BMI. Previous studies have also reported BMI as a weak predictor of neuraxial block deformity $(6,11)$. Faitot et al. (1) reported BMI along with spinal bony landmarks to be predictive of difficult spinal puncture, but a multivariate analysis demonstrated that the spinal bony landmark and not BMI was the predictor of difficulty at spinal puncture.

Theese finding is in contrast to the popular belief that patients with higher BMI kilogram/meter square $\left(\mathrm{kg} \mathrm{m}^{-2}\right)$ will have difficult epidural placement. However, there is an association between a higher BMI and a difficulty grade of spine, $94.1 \%(16 / 17)$ of patients with grade 4 spine and $72.1 \%(71 / 98)$ with grade 3 spine had a BMI of $>30(\mathrm{~kg}$ $\mathrm{m}^{-2}$ ). This finding has also been observed in a previous study where obese patients comprised the lowest proportion with good landmarks (11). As not all obese patients have difficult anatomical grade of spine, fat deposition on the back, obscuring the anatomical landmarks and not only BMI, should be taken into consideration as a predictor of difficult epidural placement. 
In another study conducted on residents with no previous experience in obstetric anaesthesia, the most reliable factors in predicting difficult epidural placement in terms of more than one attempt at catheter placement were anatomical landmarks and spinal deformity (1). However, the role of anaesthesiologist's experience remains controversial when difficulty at epidural placement is considered (11-13). In this study, we found no statistically significant difference between the number of epidural attempts and the experience level of anaesthesiologists. However, one study comparing residents in training with anaesthesiologists of varying experience found experience of the operator as an independent predictor of the first attempt success rate (13). The findings of Atallah et al. (12) also demonstrated a significant difference in the number of attempts between junior and senior providers. The lack of difference in the present study could be attributed to the fact that senior anaesthesiologists perform more challenging cases such as patients with difficult spines. This factor could have balanced the difference among the different experience levels of anaesthesiologists, as we observed that the number of epidural attempts was significantly higher in patients with difficult spines (grades 3 and 4) than in patients with easily visualised spines and palpable spinous processes and intervertebral space (grades 1 and 2). This could be the reason for the lack of statistically significant difference in the number of epidural attempts between the different experience levels of anaesthesiologists.

Achieving high patient satisfaction in labour epidural analgesia is a reflection of the quality of epidural service delivery. Previous studies have found that experiencing higher post-epidural pain score, having instrument-assisted vaginal delivery, receiving epidural catheter resiting and receiving neuraxial analgesia at a more advanced cervical dilation were the intra-partum factors that were associated with low patient satisfaction (2). However none of the previous studies have examined the association between the number of epidural attempts and patient satisfaction. One of the strengths of this study is the association between patient satisfaction and number of attempts. We observed that even though there was no difference in the pain score for patients with one attempt compared to those with more than one attempt at labour epidural insertion, the satisfaction level was low in patients with more than one attempt. Therefore, traumatic or multiple attempts at labour epidural increase patient discomfort and decrease the level of satisfaction, even with adequate pain relief during labour.

One of the limitations of this study is that we did not take into account the number of redirections of the needle or the time elapsed for the procedure that may have been required for epidural placement. Second, we did not consider the effect of loss of resistance to air or saline compared with other studies, which could have possibly affected the result (14).

\section{Conclusion}

This study conducted on 500 labour epidural procedures indicates that the most reliable method of predicting a technically difficult neuraxial block is the determination of the anatomical grade of the spine by performing an examination of the patient's vertebral column. BMI of the patient is a less reliable predictor; however, obese patients can have poor quality of anatomical landmarks depending on the distribution of fat.

It is therefore recommended that examination and documentation of anatomical grading of the spine and anatomical variants such as scoliosis should be done before the initiation of epidural placement. All epidural placements should have a mandatory section of preoperative assessment of the patients' back and the options for marking different grades of spine and any anomaly observed. It should be made mandatory for the anaesthesiologist, especially trainee residents, to seek expert help and counsel the patients whenever grades 3 and 4 levels of spine are encountered.

Ethics Committee Approval: Ethics committee approval was received for this study from the ethics committee of Aga Khan University (proposal number 3642-Ane-ERC-15).

Informed Consent: Written informed consent was obtained from patients who participated in this study.

Peer-review: Externally peer-reviewed.

Author Contributions: Concept - I.S.; Design - S.I., S.A.R.; Supervision - S.I.; Resources - S.I., S.A.R.; Materials - S.I.; Data Collection and/or Processing - S.I., S.A.R.; Analysis and/or Interpretation - S.I., S.A.R.; Literature Search - S.I.; Writing Manuscript - S.I., S.A.R.; Critical Review - S.I., S.A.R.; Other - S.I., S.A.R.

Acknowledgements: The authors wish to acknowledge the support and assistance of Seharish Sher Ali - Associate, Department of Anaesthesia, Aga Khan University, for editing the manuscript and Ms. Farzana Aziz for data collection.

Conflict of Interest: The authors have no conflicts of interest to declare.

Financial Disclosure: The authors declared that this study has received no financial support.

\section{References}

1. Faitot V, Ourchane R, Dahmani S, Magheru M, Nebout S, Gomas F, et al. An observational study of factors leading to difficulty in resident anaesthesiologists identifying the epidural space in obstetric patients. Int J Obstet Anesth 2011; 20: 124-7. [CrossRef] 
2. Tan DJA, Sultana R, Han NLR, Sia ATH, Sng BL. Investigating determinants for patient satisfaction in women receiving epidural analgesia for labour pain: a retrospective cohort study. BMC Anaesthesiol 2018; 18: 50. [GrossRef]

3. Bauer ME, Kountanis JA, Tsen LC, Greenfield ML, Mhyre JM. Risk factors for failed conversion of labor epidural analgesia to cesarean delivery anesthesia: a systematic review and meta-analysis of observational trials Int J Obstet Anesth 2012; 21 : 294-309. [CrossRef]

4. Rhee WJ, Chung GJ, Lim YH, Lee KH, Lee SC. Factors in patient dissatisfaction and refusal regarding spinal anesthesia. Korean J Anesthesiol 2010; 59: 260-4. [CrossRef]

5. Puolakka R, Haasio J, Pitkanen MT, Kallio M, Rosenberg $\mathrm{PH}$. Technical aspects and postoperative sequelae of spinal and epidural anesthesia: a prospective study of 3230 orthopedic patients. Reg Anesth Pain Med 2000; 25: 488-97. [CrossRef]

6. Ružman T, Gulam D, Haršanji Drenjančević I, Venžera-Azenić D, Ružman N, Burazin J. Factors associated with difficult neuraxial blockade. Local Reg Anesth 2014; 7: 47-52. [CrossRef]

7. Grau T, Leipold RW, Horter J, Conradi R, Martin E, Motsch J. The lumbar epidural space in pregnancy: visualization by ultrasonography.BrJ Anaesth 2001; 86: 798-804. [GrossRef]
8. Vallejo MC, Phelps AL, Singh S, Orebaugh SL, Sah N. Ultrasound decreases the failed labor epidural rate in resident trainees. Int J Obstet Anesth 2010; 19: 373-8. [GrossRef]

9. Owens E, Kasten G, Hessel E II. Spinal subarachnoid hematoma after lumbar puncture and heparinization. Anesth Analg 1986; 65: 1201-7. [CrossRef]

10. Raza D, Ismail S. Change in Mallampati class during labor: Hypertensive versus normotensive parturients. Acta Anaesthesiol Scand 2018; 62: 1086-91. [GrossRef]

11. Ellinas EH, Eastwood DC, Patel SN, Maitra-D’Cruze AM, Ebert TJ. The effect of obesity on neuraxial technique difficulty in pregnant patients: a prospective, observational study. Anesth Analg 2009; 109: 1225-31. [GrossRef]

12. Atallah MM, Demian AD, Shorrab AA. Development of a difficulty score for spinal anaesthesia. Br J Anaesth 2004; 92: 35460. [GrossRef]

13. de Filho GR, Gomes HP, da Fonseca MH, Hoffman JC, Pederneiras SG, Garcia JH. Predictors of successful neuraxial block: a prospective study. Eur J Anaesthesiol 2002; 19: 447-51. [GrossRef]

14. Agaram R, Douglas MJ, McTaggart RA, Gunka V. Inadequate pain relief with labor epidurals: a multivariate analysis of associated factors. Int J Obstet Anesth. 2009; 18: 10-4. [GrossRef] 